\title{
Nanosponges for combination drug therapy: state-of-the-art and future directions
}

\author{
Maria Tannous ${ }^{1,2}$, Francesco Trotta ${ }^{1}$ \& Roberta Cavalli*,2 \\ ${ }^{1}$ Department of Chemistry, University of Turin, via Giuria 7, 10125, Turin, Italy \\ ${ }^{2}$ Department of Drug Science \& Technology, University of Turin, via P. Giuria 9, 10125, Turin, Italy \\ *Author for correspondence: Tel.: +3901 1670 7190; Fax: +3901 1670 7687; roberta.cavalli@unito.it
}

\author{
"A combination drug therapy comprising biocompatible nanoparticles as delivery systems \\ represents a promising and challenging strategy to treat complex pathologies, such as cancer, \\ neurodegenerative disorders and severe infectious diseases."
}

First draft submitted: 7 January 2020; Accepted for publication: 10 January 2020; Published online: 20 February 2020

Keywords: co-delivery $\bullet$ combination therapy $\bullet$ cyclodextrin $\bullet$ nanosponges

Combination therapy consists of the co-administration of two or more active molecules to improve a therapeutic response in respect to that of a single drug administration. The rationale of this strategy is to exploit the occurrence of a synergistic effect. Indeed, a drug can affect the activity of another drug, producing a therapeutic benefit [1]. Moreover, co-formulations can improve the patient's compliance, reducing the number of drug administrations required. The co-delivery of anti-cancer drugs to enhance the therapeutic index and overcome multi-drug resistance in cancer treatment is a paradigmatic example of drug combination [2]. In fact, Vyxeos ${ }^{\circledR}$, a liposomal combination of daunorubicin and cytarabine contained in the same formulation, is already approved for the treatment of acute myeloid leukemia. Rituxan Hycela ${ }^{\circledR}$ (Mabthera ${ }^{\circledR}$ in Europe) and Herceptin Hylecta ${ }^{\circledR}$ are two anti-cancer marketed products containing co-formulations of antibodies designed to improve their administration.

Furthermore, the synergistic effect can be also achieved by combining small anti-cancer molecules and siRNA having different mechanisms of action, the investigation of which is still ongoing [3]. A combination drug therapy comprising biocompatible nanoparticles as delivery systems represents a promising and challenging strategy to treat complex pathologies, such as cancer, neurodegenerative disorders and severe infectious diseases. This approach can involve the co-administration of either multiple nanodelivery systems, each containing single active agents or the co-delivery of different drug molecules in the same nanocarrier. A number of examples focused on dual delivery nanocarriers have been reported in the literature, including nanoparticles, micelles, liposomes, nanotubes and silica nanospheres. Notably, nanosponge systems can play a key role for the co-administration of drugs among the multifunctional nanoparticles proposed [4].

Nanosponges are discrete solid porous nanoparticles composed of hyper-cross-linked polymers with superior drug absorption/complexation properties [5,6]. Their molecular architecture generally contains different polymer chains which can form specific microdomains suitable for the co-encapsulation of two drugs with a different chemical structure.

Polyester nanosponges have been synthetized for the co-encapsulation of tamoxifen and quercetin. The coloading was obtained by the addition of the two molecule solution on preformed nanosponge suspension. The resulting drug loading was in the same order of magnitude and the in vitro release experiments showed a similar linear release kinetics for tamoxifen and quercetin, respectively. This behavior underlines that the dual loading did not affect the individual drug release kinetics. A synergic anti-cancer effect was observed in vitro on 4T1 cell line with an increased anti-cancer activity of the two drug combination compared with that of the drug alone [7].

Interestingly, cyclodextrin-based nanosponges have attracted much research attention as drug-delivery systems for their biocompatibility, biodegradability and negligible cytotoxicity. This type of nanosponge, formed by a cluster of cross-linked cyclodextrins, showed the capability to incorporate and store either small molecules or macromolecules due to its peculiar architecture. Indeed, the nanosponge nanostructure consists of cyclodextrin 
cavities and nanochannels formed by the cross-linked network. Containing two domains with different lipophilicity, this supramolecular nanosystem can be considered a flexible platform for the delivery of either lipophilic or hydrophilic molecules. For this purpose, it is possible to select the chemical nature of the cross-linker agent (neutral, positive or negative charged) to tune the network interaction sites for drug incorporation [8,9]. Then, the cooperation of various cyclodextrin cavities present in the nanosponge network can increase the complexation capability. In addition, different types of cyclodextrins could be used as building blocks of nanosponges, producing new possibilities to accommodate drugs in the nanostructure.

Therefore, these peculiar features enable cyclodextrin-based nanosponges to be suitable for the design of codelivery drug-delivery systems. A series of benefits can be associated with this nanocarrier and some co-delivery examples have been reported in the literature.

The first advantage of nanosponges is their capability to enhance the chemical and physical stability of the incorporated drugs. The protection from degradation can be useful for designing delivery systems for nucleic acids, peptides and proteins as well as sensitive small molecules. For example, $\beta$-cycodextrin nanosponges were able to protect resveratrol from light, as previously shown [10].

The co-administration of curcumin and resveratrol, two compounds with low solubility, poor bioavailability and stability issues, has been reported. A multiple nanodelivery system formulation was studied, comprising the encapsulation of each molecule in pyromellitic dianhydride cross-linked $\beta$-cyclodextrin-based nanosponges. The two types of drug-loaded nanosponges were then dispersed in a carbomer-based hydrogel to obtain a topical coformulation. A remarkable increase of the photostability of the two compounds were observed. Furthermore, an in vitro cytotoxicity study on MCF-7 cells underlined the synergistic effect of curcumin-loaded nanosponges and resveratrol-loaded nanosponge combination [11].

Another possible approach for dual delivery consists of the sequential administration of nanoparticulate drugdelivery systems. Hariri et al. developed peptide targeted nanosponges for the co-administration of paclitaxel and camptothecin. The functionalization of nanosponges with tumor targeting peptides enhances targeting and significantly reduces tumor growth in an in vivo lung cancer model [12]. Canpotothecin is an unstable molecule sensitive to external $\mathrm{pH}$ values. In particular, this drug transforms into its inactive carboxylate form at physiological $\mathrm{pH}$ due to the opening of the lactone ring present in the molecule. As a consequence, camptothecin protection is mandatory for its in vivo administration. In previous researches we demonstrated that a carbonate nanosponge formulation was able to protect camptothecin from chemical degradation, increasing its stability at physiological $\mathrm{pH}$ [5]. Therefore, carbonate $\beta$-cyclodextrin nanosponges might be the suitable ingredients to coformulate camptothecin with other anti-cancer molecules.

The second advantage of this type of nanosponge is the capability to store liquids and gases, broadening the co-encapsulation molecule portfolio. Previous researches have demonstrated their function as reservoirs of different types of gas molecules. Intriguingly, nanosponges can work as a good reservoir of oxygen gas with a crucial role in a number of diseases. Recently, Femminò et al. showed that $\alpha$-cyclodextrin based nanosponges can work as oxygen delivery systems, being able to store and slowly release the gas with prolonged kinetics [13]. When tested in vitro, the oxygen-loaded nanosponges significantly improved the vitality of $\mathrm{H} 9 \mathrm{c} 2$ cell line in a hypoxic environment, mimicking heart failure conditions. By contrast, nitrogen-loaded $\alpha$-cyclodextrin-based nanosponges did not show any protective effect on the same cell line in hypoxic conditions. It is worth noting that tumors present hypoxic regions that hamper therapeutic treatment. The co-delivery in nanosponges of oxygen and an anti-cancer drug able to be simultaneously released in the tumor microenvironment would be a promising strategy to improve an anti-tumor therapeutic treatment.

To improve the multifunctional properties of cyclodextrin-based nanosponges it is possible to synthetize hybrid systems containing other macromolecules besides cyclodextrins. This strategy might enlarge the possibility of drug co-encapsulation and storage. Massaro et al. developed new nanosponge architecture consisting of $\beta$-cyclodextrin and calixarene covalently linked by means of triazole units. Calixarenes are supramolecular compounds formed by the condensation of phenol units and formaldehyde. They showed a good host capability in respect to a number of molecules. The association of cyclodextrins and calixarenes produced a new nanomaterial containing two different supramolecular host units in the nanostructure with complementary binding abilities, besides the presence of a triazole cross-linker network [14]. The cyclodextrin-calixarene nanosponges were studied as delivery systems for Silibinin and Quercetin, two phenolic compounds with poor water solubility and low stability. The two molecules were separately loaded and then the combination of the two systems was studied. Interestingly, the biological activities of Quercetin and Silibinin were synergistically improved by the possible concomitant cellular uptake [14]. 
Another valuable advantage of cyclodextrin-based nanosponges for co-delivery concerns the possibility to have a precise control of the drug release kinetics of the incorporated drugs by the fine tuning of the nanosponge matrix. In addition, molecularly imprinted nanosponges have been purposefully developed to obtain nanosystems with constant and sustained drug release kinetics. This technique allows the creation of polymer networks with precise template-shaped cavities to accommodate active molecules. This synthetic approach might be exploited for increasing the loading and the controlled release of co-formulated drugs.

For spatio-temporally controlled drug release, the synthesis of stimuli responsive nanosponges have been carried out. Multifunctional stimuli-sensitive nanocarriers designed for the co-delivery of two drugs might provide release of the payload in the presence of a precise external stimulus. The external triggers might favor 'on demand' drug release at the designed site, improving the therapeutic index and decreasing adverse side effects of the drugs. Stimuli-sensitive nanosponges could represent an interesting approach for combination tumor therapy. Previously, $\mathrm{pH}$ sensitive and redox responsive nanosponges have been studied for the delivery of anti-cancer drugs, showing an increased anti-tumor effect either in vitro or in vivo [15-17]. Doxorubicin incorporated into glutathione-responsive nanosponges showed a greater decrease of tumor growth in a xenograft mouse model than the free drug [15]. Glutathione/pH-responsive nanosponges loaded with strigolactone analogues showed in vitro release kinetics related to the external glutathione concentration. Moreover, the delivery of strigolactone analogues to prostate cancer cells showed a greater inhibition effect on DU145 cells with a high glutathione content than on PC3 cells with a low glutathione content [17]. These environmentally responsive nanosponges can be exploited for the co-delivery of two anti-cancer drugs to evaluate the activity of a synergistic effect in the presence of an external stimulus.

In this context, a pH-sensitive nanosponge-like architecture was obtained by the photocross-linking of 2hydroxypropil- $\beta$-cyclodextrin acrylate and two purposefully prepared chitosan derivatives for the co-delivery of paclitaxel and IL-2. The nanostructure showed a $\mathrm{pH}$ responsiveness favoring controlled drug release in the tumor microenvironment [18]. In addition, targeted nanosponges could be developed to localize drug co-administration only in specific tissues to improve their therapeutic effectiveness and decrease the administered dose. In particular, antibodies or specific ligands (i.e., folic acid, mannose) can be conjugated to the surface of co-loaded nanosponges as homing devices.

Finally, another interesting advantage could be the functionalization of co-loaded nanosponges with imaging probes, to obtain theranostic nanosystems for real-time monitoring of the drug behavior. In fact, theranostic nanosponges might allow the visualization of co-loaded nanosponge in vivo biodistribution and drug release kinetics. Recently, magnetic nanosponges were prepared by coating the surface of magnetite nanoparticles with curcumin-loaded nanosponges decorated with folic acid as a targeting agent. In vitro results showed an increased inhibitory effect of this formulation on proliferation of folate receptor-positive cancer cell lines. Furthermore, in vitro MRI imaging results demonstrated the targeting capabilities of these multifunctional nanosponges [19].

Taking into account all these advantages, much research is ongoing to optimize cyclodextrin nanosponge synthesis to obtain more tailored structures for the accommodation of different molecules in the same nanosystem. In fact, nanosponges present interesting coformulation capabilities. In particular, they could be used for the co-delivery of hydrophobic and hydrophilic drugs in the same nanosponge, when coformulation in nanocarriers has generally involved only lipophilic drugs. Moreover, cyclodextrin-based nanosponges might represent a flexible tool for the co-delivery of biologic drugs, enhancing their stability and bioavailability. Finally, they might be exploited for the coformulation of already marketed molecules to improve administration and effectiveness. Anyway, the development of a co-delivery system is more challenging than that of a monotherapy product. The manufacturing process and the analytical characterization are crucial points to be considered to obtain safe, effective and stable coformulations. Based on these premises, the combination therapy with cyclodextrin-based nanosponges can be a promising platform for future therapeutic solutions.

Financial \& competing interests disclosure

The authors have no relevant affiliations or financial involvement with any organization or entity with a financial interest in or financial conflict with the subject matter or materials discussed in the manuscript. This includes employment, consultancies, honoraria, stock ownership or options, expert testimony, grants or patents received or pending, or royalties.

No writing assistance was utilized in the production of this manuscript. 


\section{References}

1. Walvekar P, Gannimani R, Govender T. Combination drug therapy via nanocarriers against infectious diseases. Eur. J. Pharm. Sci. 127, 121-141 (2019).

2. Rawal S, Patel MM. Threatening cancer with nanoparticle aided combination oncotherapy. J. Control. Rel. 301, 76-109 (2019).

3. Huang W, Chen L, Kang L et al. Nanomedicine-based combination anticancer therapy between nucleic acids and small-molecular drugs. Adv. Drug Del. Rev. 115, 82-97 (2017).

4. Duchene D, Cavalli R, Gref R. Cyclodextrin-based polymeric nanoparticles as efficient carriers for anticancer drugs. Curr. Pharm. Biotechnol. 17(3), 248-255 (2016).

5. Swaminathan S, Cavalli R, Trotta F. Cyclodextrin-based nanosponges: a versatile platform for cancer nanotherapeutics development. Wiley Interdiscip. Rev. Nanomed. Nanobiotechnol. 8(4), 579-601 (2016).

6. Trotta F, Dianzani C, Caldera F, Mognetti B, Cavalli R. The application of nanosponges to cancer drug delivery. Exp. Opin. Drug Del. 11(6), 931-941 (2014).

7. Lockhart JN, Stevens DM, Beezer DB, Kravitz A, Harth E. Dual drug delivery of tamoxifen and quercetin: regulated metabolism for anticancer treatment with nanosponges. J. Control. Rel. 220(Pt B), 751-757 (2015).

8. Caldera F, Tannous M, Cavalli R, Zanetti M, Trotta F. Evolution of cyclodextrin nanosponges. Int. J. Pharm. 531(2), 470-479 (2017).

9. Trotta F, Zanetti M, Cavalli R. Cyclodextrin-based nanosponges as drug carriers. Beilstein J. Org. Chem. 8, 2091-2099 (2012).

10. Dhakar NK, Matencio A, Caldera F et al. Comparative evaluation of solubility, cytotoxicity and photostability studies of resveratrol and oxyresveratrol loaded nanosponges. Pharmaceutics 11(10), 545 (2019).

11. Pushpalatha R, Selvamuthukumar S, Kilimozhi D. Cyclodextrin nanosponge based hydrogel for the transdermal co-delivery of curcumin and resveratrol: development, optimization, in vitro and ex vivo evaluation. J. Drug Deliv. Sci. Technol. 52, 55-64 (2019).

12. Hariri G, Edward AD, Merrill TB, Greenbaum JM, van der Ende AE, Harth E. Sequential targeted delivery of paclitaxel and camptothecin using a cross-linked "nanosponge" network for lung cancer chemotherapy. Mol. Pharm. 11(1), 265-275 (2013).

13. Femminò S, Penna $\mathrm{C}$, Bessone $\mathrm{F}$ et al. $\alpha$-Cyclodextrin and $\alpha$-cyclodextrin polymers as oxygen nanocarriers to limit hypoxia/reoxygenation injury: implications from an in vitro model. Polymers (Basel) 10(2), 211-225 (2018).

14. Massaro $\mathrm{M}$, Cinà V, Labbozzetta $\mathrm{M}$ et al. Chemical and pharmaceutical evaluation of the relationship between triazole linkers and pore size on cyclodextrin-calixarene nanosponges used as carriers for natural drugs. RSC Adv. 6(56), 50858-50866 (2016).

15. Daga M, Ullio C, Argenziano M et al. GSH-targeted nanosponges increase doxorubicin-induced toxicity "in vitro" and "in vivo" in cancer cells with high antioxidant defenses. Free Radical Biol. Med. 97, 24-37 (2016).

16. Clemente N, Argenziano M, Gigliotti CL et al. Paclitaxel-loaded nanosponges inhibit growth and angiogenesis in melanoma cell models. Front. Pharmacol. 10, 776 (2019).

17. Argenziano M, Lombardi C, Ferrara B et al. Glutathione/pH-responsive nanosponges enhance strigolactone delivery to prostate cancer cells. Oncotarget 9(88), 35813-35829 (2018).

18. Song Q, Yin Y, Shang L et al. Tumor microenvironment responsive nanogel for the combinatorial antitumor effect of chemotherapy and immunotherapy. Nano Lett. 17(10), 6366-6375 (2017).

19. Gholibegloo E, Mortezazadeh T, Salehian F et al. Folic acid decorated magnetic nanosponge: an efficient nanosystem for targeted curcumin delivery and magnetic resonance imaging. J. Colloid Interface Sci. 556, 128-139 (2019). 